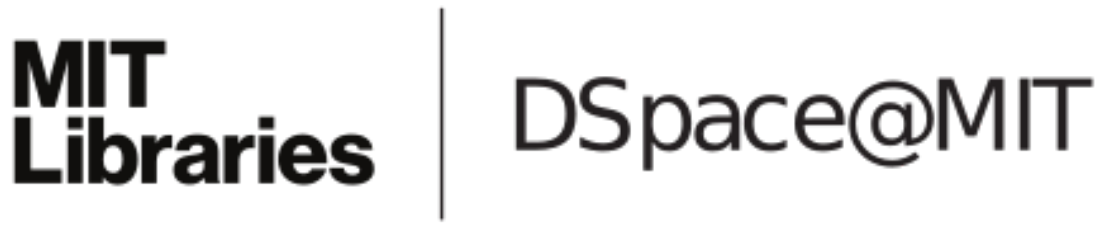

\author{
MIT Open Access Articles
}

\begin{abstract}
Electrowetting-on-dielectric actuation of a spatial and angular manipulation MEMS stage
\end{abstract}

The MIT Faculty has made this article openly available. Please share how this access benefits you. Your story matters.

Citation: Preston, Daniel J., Ariel Anders, Banafsheh Barabadi, Evelyn Tio, Yangying Zhu, DingRan Annie Dai, and Evelyn N. Wang. “Electrowetting-on-Dielectric Actuation of a Spatial and Angular Manipulation MEMS Stage." 2017 IEEE 30th International Conference on Micro Electro Mechanical Systems (MEMS) (January 2017).

As Published: http://dx.doi.org/10.1109/MEMSYS.2017.7863521

Publisher: Institute of Electrical and Electronics Engineers (IEEE)

Persistent URL: http://hdl.handle.net/1721.1/120025

Version: Author's final manuscript: final author's manuscript post peer review, without publisher's formatting or copy editing

Terms of use: Creative Commons Attribution-Noncommercial-Share Alike 


\section{ELECTROWETTING-ON-DIELECTRIC ACTUATION OF A SPATIAL AND ANGULAR MANIPULATION MEMS STAGE}

Daniel J. Preston ${ }^{1}$, Ariel Anders ${ }^{\dagger, 2}$, Banafsheh Barabadi ${ }^{\dagger, 1}$, Evelyn Tio ${ }^{1}$, Yangying $Z^{2} u^{l}$, DingRan Annie Dai ${ }^{1}$, Evelyn N. Wang ${ }^{1}$

${ }^{1}$ Department of Mech. Eng., Massachusetts Institute of Technology, Cambridge, USA ${ }^{2}$ Department of Elec. Eng. and Comp. Sci., Massachusetts Institute of Technology, Cambridge, USA

\begin{abstract}
We demonstrate a MEMS translational stage that uses electrowetting-on-dielectric (EWOD) as the actuating mechanism. Our EWOD stage is capable of linear translation with resolution of $10 \mu \mathrm{m}$ over a maximum range of $130 \mu \mathrm{m}$ and angular deflection of approximately $\pm 1^{\circ}$ while eliminating solid-solid contact. The range and resolution can be readily improved via higher base contact angle and lower contact angle hysteresis as indicated by the detailed modeling accompanying the experimental demonstration.
\end{abstract}

\section{INTRODUCTION}

Adhesion and friction during physical contact of solid components in MEMS often lead to device failure [1, 2]. Translational stages that have been fabricated with traditional silicon MEMS often face tribological concerns. Meanwhile, electrowetting, a phenomenon whereby the contact angle of a fluid can be changed with an applied voltage allowing control of droplet shape [3], has proved useful in MEMS applications ranging from fluid lenses for optical manipulation [4-7] to switches used for electrical [8, 9] and thermal control [10] and thermal management [11]. We show through modeling and experimental demonstration that EWOD has the potential to eliminate solid-solid contact during MEMS stage operation by actuating via deformable liquid droplets placed between the stage and base to achieve stage displacement as a function of applied voltage, shown schematically in Figure 1a. This operational mechanism is similar to the capillary force actuator, which relies on deformation of a liquid droplet between two solid surfaces and offers distinct advantages compared to other MEMS actuators [12]. While the actuation and dynamics [13, 14] of such devices have been explored theoretically, an experimental device without solid-solid contact has not been demonstrated, nor has angular deflection been shown [15].

\section{MODEL}

We developed an axisymmetric iterative numerical model for each of the four droplets in our device to determine the stage height as a function of the applied voltage for comparison with experimental results obtained from the working device. First, the contact angle at the EWOD base was determined with the Lippman-Young equation as a function of the intrinsic contact angle and applied voltage as well as the capacitive properties of the dielectric layer. Then, the droplet curvature in the system
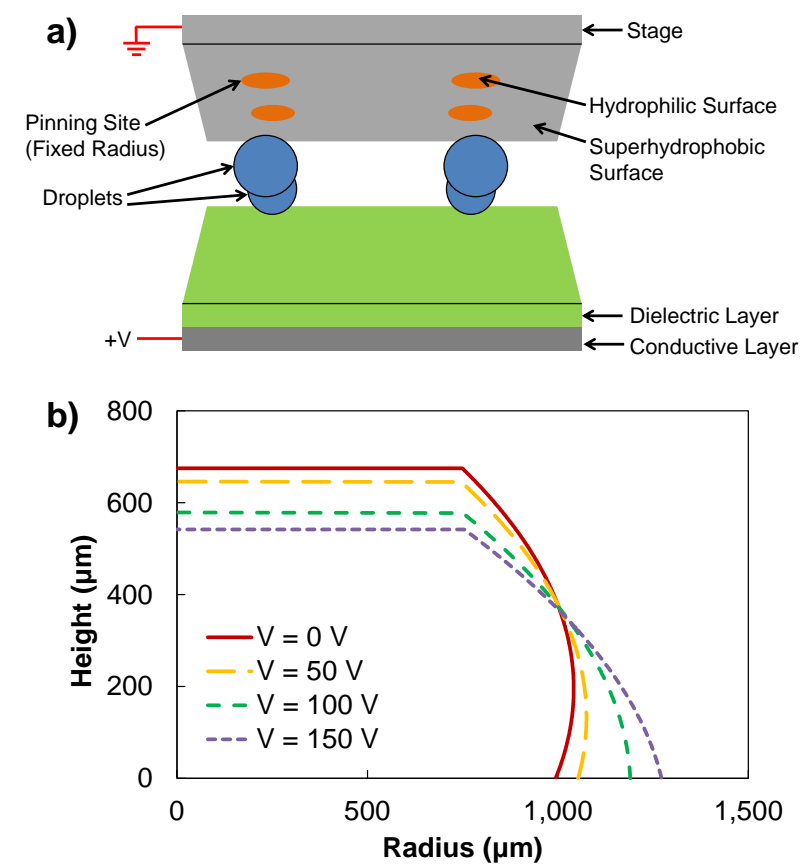

Figure 1: (a) The electrowetting-actuated stage is shown schematically. The tops of the water droplets contact the underside of the stage at electrically conducting hydrophilic copper pinning sites surrounded by a superhydrophobic surface of functionalized $\mathrm{CuO}$ nanoblades, and the bottoms of the droplets contact the insulated electrowetting-ondielectric base. (b) The expected stage height is determined by our axisymmetric model. Model droplet profiles as a function of applied voltage are shown for a $2 \mu \mathrm{L}$ droplet and base Young contact angle of $110^{\circ}$. Contact with the region of fixed radius on the underside of the stage corresponds to the flat regions at the top of the droplet profiles.

was calculated as a function of the internal Laplace pressure, which was determined at the top of the droplet (underside of the stage) by summing one quarter of the stage weight with the surface tension force pulling downwards along the pinned three-phase contact line at the perimeter of the droplet and then dividing that quantity by the fixed top contact area. Note that the droplet curvature relies on the initially unknown droplet contact angle at the underside of the stage; as such, an iterative solution was implemented. The complete droplet profile was determined numerically under the constant curvature constraint by iterating until convergence. The model was used to determine the profiles of droplets under different applied voltages, shown in Figure 
1b. The flat region at the top of each profile is the contact with a pinning site on the bottom of the stage, where the constant radius over different applied voltages is consistent with the physical picture. The contact angle at this pinned region varies, as does the radius of the base on the EWOD surface, both of which are expected.

\section{EXPERIMENT}

We fabricated the stage by first growing rough copper oxide $(\mathrm{CuO})$ nanoblades on copper foil following a common procedure detailed in the literature [16] and then functionalizing the $\mathrm{CuO}$ with a monolayer of trichloro $(1 \mathrm{H}, 1 \mathrm{H}, 2 \mathrm{H}, 2 \mathrm{H}$-perfluorooctyl)silane (SigmaAldrich) to form a superhydrophobic surface. The advancing and receding contact angles of the superhydrophobic $\mathrm{CuO}$ were $172^{\circ} \pm 3^{\circ}$ and $168^{\circ} \pm 3^{\circ}$, respectively, as measured with a microgoniometer (MCA-3, Kyowa). The pinning sites were subsequently formed on the underside of the stage by milling away the $\mathrm{CuO}$ to a negligible depth using an end mill with a diameter of approximately $1.5 \mathrm{~mm}$, thereby exposing the hydrophilic [17] copper and forming a liquid pinning site at the junction of the hydrophilic and superhydrophobic regions to fix the top radius of the droplets. Finally, to establish a nonconstraining electrical connection with the stage, a copper wire was soldered in a vertical orientation to a tab at the corner of the stage in order to attach to a sliding attachment mounted above the stage. The total stage mass was $0.090 \mathrm{~g}$.

Indium-tin-oxide-coated glass slides with resistivity of $10 \Omega /$ sq were used as the conductive substrate for the EWOD base. The slides were solvent and plasma cleaned, then coated with a $4 \mu \mathrm{m}$ thick parylene-C layer (VSI Parylene, precision $\pm 1 \mu \mathrm{m}$ ) with dielectric strength of $22,000 \mathrm{~V} / \mathrm{m}$ and relative permittivity of $\varepsilon r \approx 3$. The slides were subsequently coated with a sub-micron coating of Teflon aqueous fluoropolymer (AF) as detailed in past work [18] in order to promote a high intrinsic contact angle $\left(\approx 116^{\circ}\right)$ with low contact angle hysteresis. The water

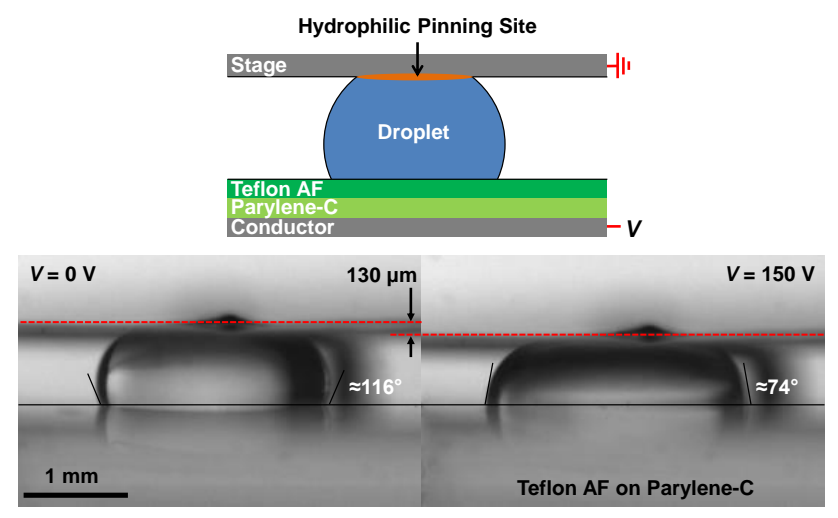

Figure 2: Schematic and photos of the initial neutral state and the stage vertical translation at an applied voltage of $150 \mathrm{~V}$, which resulted in a stage deflection of $130 \mu \mathrm{m}$ on using the Teflon AF-coated base surface compared to the initial neutral position (zoomed in to one droplet). contact angle at varying applied voltages on the EWOD base was characterized by applying voltage through a copper wire electrode inserted into a single $2 \mu \mathrm{L}$ droplet of $0.1 \mathrm{mM} \mathrm{KCl}$ solution in water resting on the EWOD base. The EWOD base was electrically grounded with copper alligator clips penetrating through the dielectric coating to the ITO and subsequently mounted on the stage in front of the high-speed camera. The voltage was varied up to $150 \mathrm{~V}$, and the contact angle was in excellent agreement with the Lippmann-Young prediction until the saturation voltage, which occurred at a contact angle of $74^{\circ}$ for the Teflon AF coated parylene-C surface.

The experimental setup consisted of a function generator (AFG 3101, Tektronix) passed through a 400x voltage amplifier (A800, FLC Electronics) with the positive lead wired to the EWOD base and the negative lead attached to the stage via the sliding electrical connection to allow free translation in the vertical z-direction. The stage provided direct electrical connection to the droplets through the conductive hydrophilic copper circles on its underside.

The device was both front- and back-lit for high-speed video capture (Phantom v7.1, Vision Research) from 500 to 10,000 frames per second as the experiment was conducted. Four droplets of $0.1 \mathrm{mM} \mathrm{KCl}$ solution in water with a volume of $2 \mu \mathrm{L}$ were carefully pipetted onto the pinning sites on the underside of the stage, which was then inverted and placed onto the EWOD base (the pinned droplets did not fall from the stage). Finally, the stage sliding electrical connection was attached for initial characterization.

Images during a typical experiment were captured in Figure 2. At an applied voltage of $150 \mathrm{~V}$, the contact angle decreased from $116^{\circ}$ to $74^{\circ}$ on the Teflon AF coated EWOD base. This change in contact angle caused the droplets to spread while the volume remained constant and thus resulted in a decrease in stage height as predicted by the model. Tests at intermediate voltages in Figure 3 (black circles indicate results with the sliding electrical connection) show good agreement with the model prediction for a $2 \mu \mathrm{L}$ droplet generated by the iterative solution.

\section{RESULTS}

To eliminate solid-solid contact and the accompanying stiction and tribological concerns, the stage was reconfigured to remove the requirement for the sliding electrical connection. This was achieved by separating the Teflon AF coated EWOD base into two electrically insulated components, each holding two droplets (Figure 3). Then, voltage was applied from one insulated section of the EWOD base to the other, forming a circuit comprised of two capacitors (the dielectric regions at the base of the droplets on each of the insulated EWOD base sections). Since each of these series capacitors carry half of the applied voltage, twice the voltage required in the previous configuration is required for the same stage deflection. The stage deflection in this configuration was experimentally demonstrated to be equivalent to the previous (wired) configuration and in good agreement with the model, as shown in Figure 4b. 

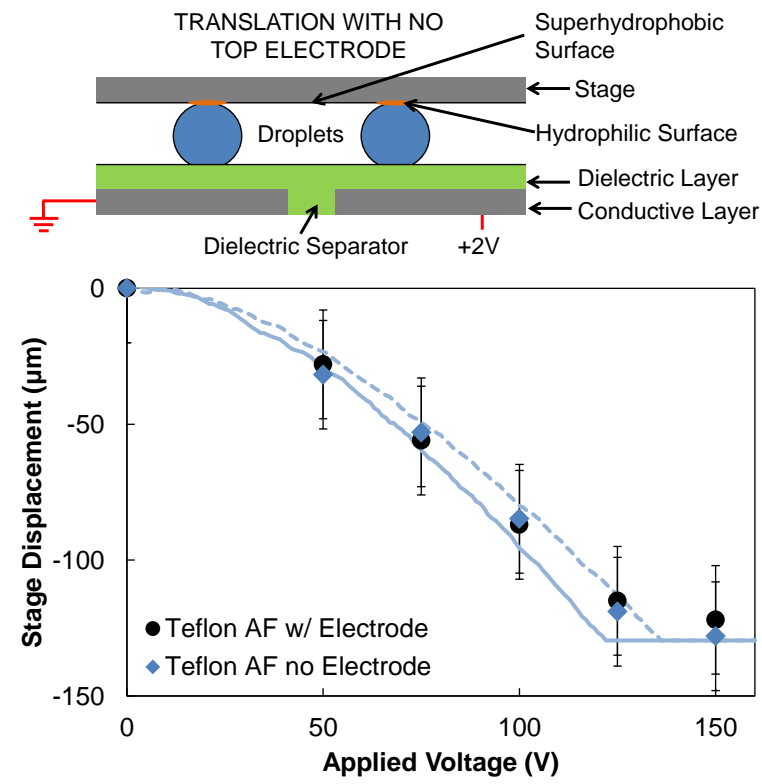

Figure 3: Elimination of the stage electrode is achieved by applying the voltage to two droplets in series from the base with a dielectric separator between two pairs of droplets and provides the same stage deflection as a function of voltage as the wired version, both of which are in excellent agreement with the model (solid and dashed line indicate lower- and upper-bound model predictions).

Additionally, the stage can provide angular deflection. The configuration was further modified to keep the electrically separated EWOD base but once again include the stage sliding electrical connection, which was grounded (Figure 4). When a voltage is applied to either insulated section of the EWOD base, that side of the stage is displaced downwards while the other side remains unperturbed, resulting in angular deflection. To test this configuration, we constructed a varying voltage that first actuated one side of the stage, and then actuated the other side of the stage. The function generator/amplifier output was set to increase from $0 \mathrm{~V}$ to $150 \mathrm{~V}$ and then decrease back to $0 \mathrm{~V}$ repeatedly as a sine wave with amplitude $75 \mathrm{~V}$, offset $+75 \mathrm{~V}$, and period $2 \mathrm{sec}$. This signal was followed by a microcontroller (UNO R3, Arduino) which used a motor shield (L298P, Arduino) to switch relays (7266K64, McMaster-Carr) that alternated the applied voltage between the two sides of the EWOD base each time the signal bottomed out at $0 \mathrm{~V}$, leaving the non-active side of the EWOD base at $0 \mathrm{~V}$. The result of the signal applied to this configuration is shown in Figure 4, where each side of the stage deflected by approximately $130 \mu \mathrm{m}$ when the voltage was applied (diamond and square symbols), in agreement with the uniform vertical stage displacement demonstrated previously, and the stage angular displacement varied from approximately $-1^{\circ}$ to $+1^{\circ}$ (hollow circular symbols).

Combining the two modified configurations above could yield an angular deflection stage that does not require any solid-solid contact (no stage electrode connection).
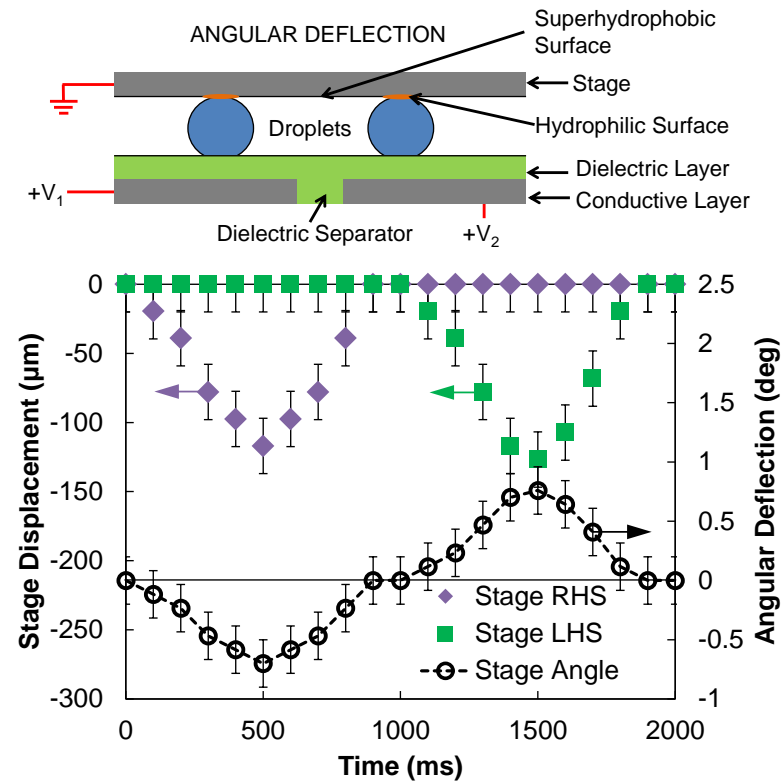

Figure 4: Angular deflection of the stage, achieved by applying a sinusoidal signal with differing voltages to the two isolated sections of the base while the stage is grounded, reached approximately one degree in the current configuration. These two approaches can be combined for angular deflection without a direct electrical connection to the stage, thus providing angular deflection without solidsolid contact.

This is possible by separating the EWOD base into four electrically isolated sections, one for each droplet, and then essentially controlling the deflection of two adjacent droplets by applying a voltage across the EWOD base beneath those droplets. Such a configuration would also allow angular deflection along any axis of rotation within the plane of the stage. A further expansion of this concept could build on past work in which the EWOD surface was separated into an array of isolated electrodes actuated separately to induce lateral droplet motion [19-21]. Operation of the stage described in the present work on such an array of electrodes could allow for lateral as well as vertical translation.

\section{CONCLUSIONS}

This work shows a MEMS vertical translation stage that uses EWOD as the actuating mechanism. The EWOD stage was capable of linear spatial manipulation with resolution of $10 \mu \mathrm{m}$ over a maximum range of $130 \mu \mathrm{m}$, which can be readily improved in future device generations using the validated model developed in the present work as a guide. Specifically, a higher intrinsic contact angle on the EWOD base improves absolute range, and reduction of contact angle hysteresis, possibly by addition of a lubricant to the surface [22, 23] or careful control of contaminants [24], will increase resolution. In addition, angular deflection of approximately $\pm 1^{\circ}$ was demonstrated, and the maximum range and angular deflection are comparable to an alternate 
type of actuator, piezoelectrics. The capability to operate the stage without any solid-solid contact makes this a desirable potential solution to stiction and tribology concerns for improvement of applications in micro-optics, actuators, and other MEMS.

\section{ACKNOWLEDGEMENTS}

We thank Professors John W. M. Bush and Michael Cima at MIT for fruitful discussions regarding the work. We gratefully acknowledge funding support from the Office of Naval Research (ONR) with Dr. Mark Spector as program manager. D. J. Preston acknowledges funding received from the National Science Foundation (NSF) Graduate Research Fellowship under Grant No. 1122374. We also acknowledge the support from the NSF through the Major Research Instrumentation Grant for Rapid Response Research (MRI-RAPID).

\section{REFERENCES}

[1] S. K. Sinha, et al., Nano-tribology and materials in MEMS: Springer Berlin Heidelberg, 2013.

[2] B. Bhushan, "Adhesion and stiction: Mechanisms, measurement techniques, and methods for reduction," Journal of Vacuum Science \& Technology B, vol. 21, pp. 2262-2296, Nov-Dec 2003.

[3] M. G. Lippmann, "Relation entre les phénomènes électriques et capillaires," Ann. Chim. Phys., vol. 5, 1875 .

[4] T. Krupenkin, et al., "Tunable liquid microlens," Applied Physics Letters, vol. 82, pp. 316-318, Jan 202003.

[5] X. F. Zeng and H. R. Jiang, "Liquid tunable microlenses based on MEMS techniques," Journal of Physics D-Applied Physics, vol. 46, Aug 14 2013.

[6] F. Krogmann, et al., "A MEMS-based variable micro-lens system," Journal of Optics A - Pure and Applied Optics, vol. 8, pp. S330-S336, Jul 2006.

[7] M. A. Bucaro, et al., "Tunable Liquid Optics: Electrowetting-Controlled Liquid Mirrors Based on Self-Assembled Janus Tiles," Langmuir, vol. 25, pp. 3876-3879, Mar 172009.

[8] P. Sen and C. J. Kim, "A fast liquid-metal droplet microswitch using EWOD-driven contact-line sliding," Journal of Microelectromechanical Systems, vol. 18, pp. 174-185, Feb 2009.

[9] P. Sen and C. J. Kim, "A Liquid-Metal Rf Mems Switch with Dc-to-40 Ghz Performance," Ieee 22nd International Conference on Micro Electro Mechanical Systems (Mems 2009), pp. 904-907, 2009.

[10] J. Gong, et al., "Thermal switches based on coplanar EWOD for satellite thermal control," Mems 2008: 21 st Ieee International Conference on Micro Electro Mechanical Systems, Technical Digest, pp. 848-851, 2008.
[11] A. L. R. McLanahan, et al., "A Dielectric Liquid Contact Thermal Switch with Electrowetting Actuation," Proceedings of the Asme International Mechanical Engineering Congress and Exposition - 2010, Vol 7, Pts a and B, pp. 61-66, 2012.

[12] C. R. Knospe and S. A. Nezamoddini, "Capillary Force Actuation," J. Micro-Nano Mech., vol. 5, pp. 57-68, 2009

[13] C. R. Knospe, "Capillary Force Actuation: A Mechatronic Perspective," Control Technologies for Emerging Micro and Nanoscale Systems, vol. 413, pp. 201-218, 2011.

[14] C. R. Knospe and H. Haj-Hariri, "Capillary force actuators: Modeling, dynamics, and equilibria," Mechatronics, vol. 22, pp. 251-256, Apr 2012.

[15] H. H. Wang, et al., "The Capillary Force Actuator: Design, Fabrication and Characterization," 2012 Ieee 25th International Conference on Micro Electro Mechanical Systems (Mems), 2012.

[16] N. Miljkovic, et al., "Electrostatic charging of jumping droplets," Nature Communications, vol. 4, Sep 2013.

[17] D. J. Preston, et al., "Scalable Graphene Coatings for Enhanced Condensation Heat Transfer," Nano Letters, vol. 15, pp. 2902-2909, May 2015.

[18] A. Cavalli, et al., "Electrically induced drop detachment and ejection," Physics of Fluids, vol. 28, Feb 2016.

[19] Q. Ni, et al., "Open-loop electrowetting actuation with micro-stepping," Sensors and Actuators aPhysical, vol. 247, pp. 579-586, Aug 152016.

[20] H. L. Ricks-Laskoski, et al., "EWOD-Driven Translational Movement of a Liquid Polyelectrolyte Droplet," Journal of Applied Polymer Science, vol. 110, pp. 3865-3870, Dec 15 2008.

[21] C. G. Cooney, et al., "Electrowetting droplet microfluidics on a single planar surface," Microfluidics and Nanofluidics, vol. 2, pp. 435446, Sep 2006.

[22] A. Lafuma and D. Quere, "Slippery pre-suffused surfaces," Epl, vol. 96, Dec 2011.

[23] T. S. Wong, et al., "Bioinspired self-repairing slippery surfaces with pressure-stable omniphobicity," Nature, vol. 477, pp. 443-447, Sep 222011.

[24] D. J. Preston, et al., "Effect of hydrocarbon adsorption on the wettability of rare earth oxide ceramics," Applied Physics Letters, vol. 105, Jul 7 2014.

\section{CONTACT}

*D.J. Preston; tel: +1-617-324-2087; email: dpreston@mit.edu

${ }^{\dagger}$ Denotes equal contribution 